\title{
Endogenous shifts of attention cause distortions in the perception of space: \\ Reviewing and examining the Attentional Repulsion Effect
}

Rebecca K. Lawrence, Dana Kulzhabayeva, and Jay Pratt

The University of Toronto

- The Final Published version of this manuscript is as follows: Lawrence, R. K., Kulzhabayeva, D., \& Pratt, J. (2020). Endogenous shifts of attention cause distortions in the perception of space: Reviewing and examining the attentional repulsion effect. Visual Cognition, 1-19.

https://doi.org/10.1080/13506285.2020.1793438

Corresponding Author: Rebecca K. Lawrence

Address: School of Applied Psychology,

Griffith University, Gold Coast Campus

Email: rebecca.lawrence $@$ griffith.edu.au 


\begin{abstract}
The functioning of spatial attention and its effects on visual processing are typically studied using chronometric and accuracy measures of behavior. However, as an alternative to these measures, a growing body of literature has studied the attentional repulsion effect (ARE). Simply put, when attention is focused on one location in the visual field, stimuli appearing nearby the attended location are perceived as being located further away from the attended location than they physically are. The ARE is particularly compelling, as it is best explained by considering the receptive field properties of visual cells, thus allowing for more direct comparisons between behavior and neural functioning. Nonetheless, the majority of research testing the ARE has manipulated spatial attention exogenously. Furthermore, for studies that have explored endogenous attention and the ARE, empirical evidence is conflicting. As such, the aim of the current study was to address this inconsistency by testing the effect of voluntary attention on spatial repulsion using an optimal operationalization of endogenous attention. Specifically, centrally presented, highly informative double-cues were used to shift attention, and placeholders were included in the visual display to help anchor attention. Under these optimal conditions, we observed strong spatial repulsion effects when attention was shifted endogenously. These results provide strong evidence that voluntary deployments of attention can cause perceptual distortions of space.
\end{abstract}

Key Words: Attention Repulsion, Endogenous Attention, Exogenous Attention 


\section{Endogenous shifts of attention can cause distortions in the perception of space: Reviewing and examining the Attentional Repulsion Effect}

The visual world contains numerous and complex sensory signals that humans must parse out into meaningful units for perception and action. Therefore, for meaningful processing, spatial attention is utilized to select relevant regions of space (Carrasco, 2011; Desimone \& Duncan, 1995; Posner, 1980; Posner \& Cohen, 1984). Is has long been thought that spatial attention can be guided to specific regions of space either endogenously or exogenously (Carrasco, 2011; Jonides, 1981; Müller \& Rabbitt, 1989; Nakayama \& Mackeben, 1989). Endogenous attention refers to intentional deployments of spatial attention that occur in the absence of any peripheral events. For example, when reading, attentional resources are voluntarily shifted across the static letters and words on a page to decipher meaning. In contrast, exogenous attention refers to unintentional deployments of spatial attention that occur in the absence of any task-relevant or goal-directed strategies. For instance, during a thunderstorm, a bright flash of lightning might automatically attract attention.

In the lab, the effects of visuospatial attention on visual processing are often studied using variations of the Posner cueing paradigm (Posner, 1980; Posner \& Cohen, 1984; Posner, Rafal, Choate, \& Vaughan, 1985). At the beginning of a trial, participants maintain their gaze on a central area of the visual field. Next, spatial attention is shifted to one of two locations using peripheral or central cues. Peripheral cues consist of any physical event away from fixation (often the brief onset and offset of an object) and are thought to rapidly pull attention towards the cued location in space exogenously, making it a fast-acting process peaking at around 100-200ms. In contrast, central cues are typically symbolic and informative, aiming to push attention to a particular 
location endogenously. Because participants have to interpret the cue, and then shift attention, it is thought that endogenous attention is a relatively slow process which is typically deployed in approximately 300ms (Carrasco, 2011; Jonides, 1981; Müller \& Rabbitt, 1989; Nakayama \& Mackeben, 1989). After the cue presentation, a target appears in at the cued or uncued location (typically the onset of an object), and participants are asked to detect or discriminate the target as quickly and accurately as possible. In both cases, improved accuracy and faster responding at the cued location indicates an attentional shift to the region in the visual field indicated by the cue.

The majority of research studies exploring the dynamics of visuospatial attention and visual perception use accuracy and reaction time (RT) measures (e.g., Giordano, McElree, \& Carrasco, 2009; Nakayama \& Mackeben, 1989; Posner, 1980). There is, however, a small but active literature that has demonstrated that focused attention can alter the perception of space (e.g., Kosovicheva, Fortenbaugh, \& Robertson, 2010; Pratt \& Turk-Browne, 2003; Suzuki \& Cavanagh, 1997). This striking phenomenon is labeled the attentional repulsion effect (ARE) by Suzuki and Cavanagh (1997), who were the first to discover it. Figure 1 displays the basic paradigm. Following a period of fixation, two diagonally separated peripheral luminance cues are presented. These cues are assumed to pull attention to opposite corners of the visual field. Next, participants are asked to complete a Vernier acuity task presented in central vision. Usually, one line is presented above a fixation cross, and one line is presented below. The lines are slightly vertically offset and parallel and participants report the direction of offset using a keypress (e.g., is the top line offset to the left or to the right?). Changes in perceived space are calculated by determining the absolute physical offset required for the Vernier lines to appear aligned using a thresholding procedure (e.g., Suzuki \& Cavanagh, 1997). Alternatively, some researchers have compared the total number of 'left' (or 'right' ) responses made by participants for each line and cue location 
(e.g., Pratt \& Turk -Browne, 2003). Critically, following a cue, participants perceive the lines as being shifted away from the cued location. Cues appearing in the top left and bottom right location of the visual field lead people to perceive the Vernier lines as being offset in a clockwise direction, whereas cues appearing in the top right and bottom left corners of the visual field lead people to perceive the Vernier lines as being offset in a counterclockwise direction. Thus, it appears that attention can qualitatively shift the perception of object locations in the visual field.

The ARE has important implications for our understanding of the effects of visuospatial attention on visual perception. Specifically, the ARE can be understood by considering the properties of visual cell receptive fields in the brain (Suzuki \& Cavanagh, 1997). A receptive field refers to the location out in space that a visual neuron is most responsive to. When a target appears in a particular location in the visual field, cells tuned to respond to this location will fire, transmitting information through the visual system for perception. Populations of cells can provide information about changes across in the visual environment, and help provide a coherent percept of the world. Receptive fields are organized topographically, such that cells next to one another process adjacent regions of the visual field. Furthermore, receptive fields are larger at father eccentricities, as well as higher levels of the visual hierarchy, leading to improved visual acuity in central compared to peripheral vision (Hubel \& Wiesel, 1962, 1968; Wiesel \& Hubel, 1966; Zeki, 1980). 


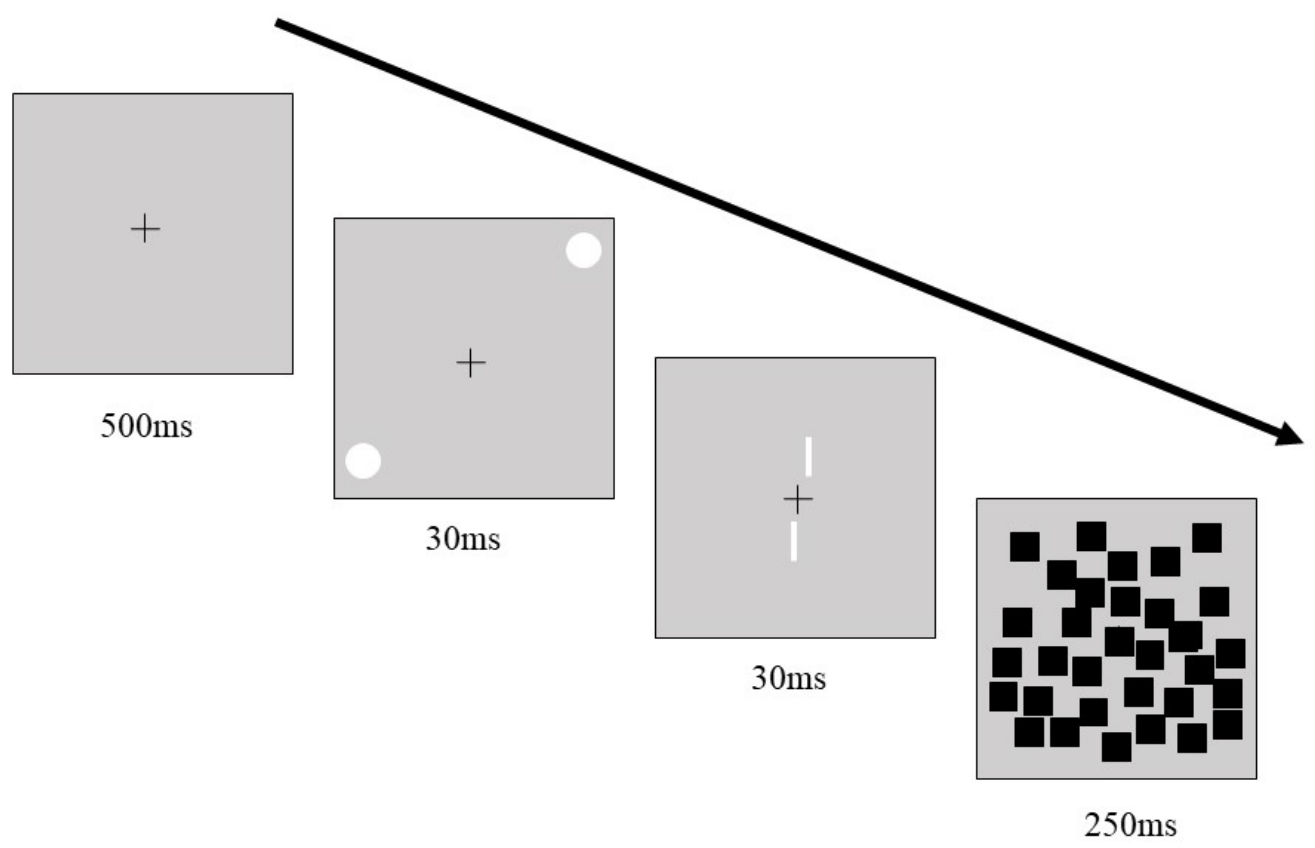

Figure 1. Example of a typical ARE paradigm. Following a period of fixation, two white circles are briefly flashed for $30 \mathrm{~ms}$ at opposite corners of the visual field. Following this, participants complete a Vernier alignment task presented in central vision. Following the Vernier line presentation, a mask is displayed to remove the potential influence of visual aftereffects.

Suzuki and Cavanagh (1997) suggested that there are at least three ways visuospatial attention might influence receptive fields to produce the ARE. Firstly, attentional cues might cause receptive cells to shift. Specifically, receptive fields of cells nearby the attended location might get dragged towards the cue. This shift would lead to an overlap of receptive fields at the cued location, increase sampling, and thus improve visual resolution where attention is focused. However, because receptive fields would have shifted away from their original location, there 
would be lower sampling at the location in space that receptive cells had shifted from (i.e., a shift in the population response of the cells towards the cued location, and away from their previous location). A consequence of this is that the perception of space may be distorted at these nearby locations, resulting in repulsion.

Supporting this first possibility is a growing neurophysiological literature demonstrating that attention can cause shifts in the receptive field profiles of individual cells (for a review, see Anton-Erxleben \& Carrasco, 2013). For example, Connor, Gallant, Preddie, and Van Essen (1996) measured neuronal responses in the visual cells of monkeys while they covertly (without eye movements) attended to targets in the visual field. Overall, the receptive fields of $49 \%$ of the neurons recorded moved in the direction of the attended location. Likewise, Womelsdorf, AntonErxleben, Pieper, and Treue (2006) showed that the receptive fields of visual area MT in rhesus monkeys shifted towards attended locations in the visual field. Finally, using high-resolution functional magnetic resonance imaging, Klein, Harvey, and Dumoulin (2014) demonstrated that in humans, population receptive fields' shift towards the attended location during a covert attention task, while de Haas, Schwarzkopf, Anderson and Rees (2014) demonstrated that perceptual load also affects responses of population receptive fields in the visual cortex. Taken together, this provides a physiologically plausible mechanism to account for the ARE where shifts in visual cell receptive fields lead to attentional repulsion in central vision.

Suzuki and Cavanagh's (1997) second possibility was based on the notion that the ARE could occur due to receptive field size changes at the attended location. In particular, receptive fields might shrink or expand to the size of the cued region. In turn, smaller receptive fields would improve visual resolution, by allowing for fine-grained processing of luminance changes across space. However, the shrinking of receptive fields at the cued location would also lead to objects 
appearing nearby the cued location to fall outside of the receptive field of these cells, which could, in turn, cause a repulsion effect. This would be seen via a shift in the population response of receptive fields towards the attended location (Suzuki \& Cavanagh, 1997).

Similar to receptive field shifting, there is neurophysiological evidence that covert attention can alter receptive field size. In a primate study, Anton-Erxleben, Stephan, and Treue (2009) measured changes in the size of MT receptive fields when monkeys attended to locations falling inside and outside of visual cell receptive fields. When the monkeys attended to locations inside of a receptive field, the receptive field contracted. Conversely, when attending to locations outside a receptive field, it expanded to encompass the attended location. Similarly, Fischer and Whitney (2009) found that populations of neurons in V1 have sharper response profiles under focused spatial attention, which indirectly suggests that attention can contract visual cell receptive fields.

The final possible explanation brought forward by Suzuki and Cavanagh (1997) is that the ARE occurs due to surround-suppression. Here, it is proposed that neuronal activity for cells nearby the attended location is reduced. The reduced activity would lower noise from cells in surrounding locations, leading to sharper responses at cued locations, but decreased responses at nearby locations. Critically, this lowered activity could result in a repulsion effect. Consistent with the suppression hypothesis, Hopf et al. (2006) demonstrated that although neuronal activity is enhanced at the attended location during a visual search task, a region of suppression surrounding the attended area also exists. In this study, participants were shown a series of 9 Landolt Cs, one of which differed in color and served as a pop-out target. Participants discriminated the orientation of the odd colored Landolt, while neural activity from the visual cortex was recorded using highresolution magnetoencephalography. Changes in activation localized broadly to the visual cortex 
indicated a neural suppression around the attended region. Thus, as well as enhancing perception at the attended location, it appears that inhibitory mechanisms might influence processing at nearby locations in the visual field.

Despite its far-reaching implications for our understanding of visuospatial attention by connecting receptive field activity directly to attentional orienting, since its discovery, an unexpectedly small literature exploring the ARE has emerged (However, for other approaches to understanding attention and receptive field functioning, see Carrasco \& Yeshurun, 2009; Carrasco, 2011; Anton-Erxleben \& Carrasco, 2013; Carrasco \& Barbot, 2015). Key papers related to the ARE are summarized in Table 1. Generally speaking, these studies have shown that the ARE emerges in a variety of situations. For example, an early study conducted by Pratt and TurkBrowne (2003) suggests that the ARE occurs for object perception and perception for action (Goodale \& Milner, 1992). Later, Gozli and Pratt (2012) demonstrated that the ARE is impervious to attentional control set manipulations (Folk, Remington \& Johnston, 1992). Finally, the ARE has emerged for both visual and lateralized sound cues (Arnott \& Goodale, 2006), under both monocular and binocular viewing conditions (DiGiacomo \& Pratt, 2012), and even without conscious awareness (Au, Ono, \& Watanabe, 2013). Together, these studies suggest that the ARE is a largely robust phenomenon.

The majority of research showing the viability of the ARE has, however, used briefly presented, peripheral non-predictive cues to shift spatial attention. Thus, most conclusions related to the ARE may be specific to cueing paradigms where attention is deployed exogenously. In contrast, less research has tested the effect of endogenous attention on spatial repulsion. This imbalance in the literature is surprising, as the effects of endogenous versus exogenous cueing using chronometric and accuracy measures of visual processing at the attended location have been 
extensively studied in the literature. Furthermore, endogenous and exogenous attention are known to have dissociable effects on visual processing (e.g. Barbot, Landy, \& Carrasco, 2012; Carrasco, 2011; Chica et al., 2014; Grubb, White, Heeger, \& Carrasco, 2015; Hein, Rolke, \& Ulrich, 2006; Jonides, 1981; Posner \& Cohen, 1984; Prinzmetal, McCool, \& Park, 2005; Yeshurun, Montagna, \& Carrasco, 2008). For instance, some researchers have found volitional attention to improve temporal resolution (detecting visual changes across time) but automatically deployed attention to impair it (Hein et al., 2006; Yeshurun \& Levy, 2003). Finally, studies exploring endogenous and exogenous attention have shown that the two processes have some unique effects on electrophysiological responses in the brain (e.g., Hopfinger \& West, 2006). However, it is important to note that there is a degree of overlap in the two networks and that the two networks interact with one another (Corbetta \& Shulman, 2002; Dugué, Merriam, Heeger \& Carrasco, 2017; Hopfinger \& West, 2006; Kincade et al., 2005; Peelen, Heslenfeld, \& Theeuwes, 2004; Macaluso \& Doricchi, 2013). Nonetheless, it is reasonable to postulate that endogenous and exogenous shifting might differentially affect attentional repulsion.

Even more problematic is that for the literature that does exist, there is mixed empirical evidence of studies on endogenous attention and the ARE. Specifically, while some research has observed reliable AREs (Cutrone, Heeger, \& Carrasco, 2018; Suzuki \& Cavanagh, 1997), others have found endogenous cueing to have minimal effects on repulsion (Petersson, Hilchey, \& Pratt, 2019). Understanding this contradictory evidence is crucial for our conceptualization of the ARE, as research to date would predict similar AREs for both endogenous and exogenous cueing. That is, spatial attention should cause changes in receptive fields, regardless of how attention was deployed initially. As such, the aim of the current study is to better understand under which conditions endogenous repulsion can be observed. Firstly, a brief review of the concepts of 
endogenous and exogenous attention is canvassed to provide a clear operationalization of what the two terms refer to in the attentional cueing literature. Next, these operational criteria are applied to the current studies claiming to test endogenous attention and the ARE. Finally, we present data from an empirical experiment that tests the effects of purely endogenous attention on spatial repulsion. Ultimately, we show that endogenous attentional deployment results in reliable repulsion effects while resolving the current ambiguity present in the literature. 
Table 1. Summary of key ARE papers

Study Summary

\begin{tabular}{ll}
\hline Arnott and Goodale & Audio cues lateralized to the left or right of a participant can distort the perception of space. This finding suggests \\
$(2006)$ & that sound processing can interfere with the receptive field properties of visual cells.
\end{tabular}

$\mathrm{Au}$, Ono \& Watantabe. Demonstrated that the ARE can occur without participants consciously processing attentional cues, although the effects were weaker than those typically observed during conscious processing. During the study, peripheral cues were visually masked, ensuring their location was invisible to participants.

Baumeler and Born Explored hemispheric influences in the ARE. Attention was deployed to the top left or right corners of a computer (2019) screen. Participants then completed a Vernier task, where the bottom line was presented at 0 eccentricity, and the top line was presented to the left or to the right of $0^{\circ}$. The magnitude of the ARE was highest when the cue and target appeared on the same side of the screen (hemifield).

Chien, Ono, and This study tested whether the ARE and attentional attraction effects are governed by similar cognitive mechanisms. Watanabe (2011) In particular, the authors suggested that attentional attraction effects are driven by shifts of attention, whereas repulsion effects are the result of static attention at one location. 
Cutrone et al. (2018) This study assessed the effects of endogenous attention on the ARE using a rapid serial visual presentation (RSVP) task and a Vernier line task. Participants attended to two diagonally offset locations indicated by centrally presented predictive cues and searched for a target in the RSVP stimuli presented peripherally. If the target was not present in the RSVP task, they then completed a centrally presented Vernier task. An ARE was observed.

DiGiacomo and Pratt Demonstrated that the size of the ARE was similar for monocular and binocular viewing conditions, while also finding little evidence of interocular transfer of the ARE. The authors concluded that the ARE emerges during early visual processing.

Gozli and Pratt (2012) Attentional control sets do not interfere with the ARE. Participants completed a Vernier alignment task consisting of two colored lines (e.g., red). The color of the lines formed the participant's attentional control set. Attention was manipulated using peripheral cues that were either the same or a different color to the control set. The ARE emerged in both conditions. 
Klein, Paffen, Pas, and This study tested the magnitude of the ARE as a function of inferred receptive field sizes at various stimulus Dumoulin (2016) eccentricities and for different spatial frequencies. Spatial attention altered the perceived distance between two Gabors in the visual field. The effect was modulated by eccentricity, where greater eccentricities led to more substantial repulsion effects. In contrast, the spatial frequency of the Gabors did not alter the magnitude of the ARE.

Kosovicheva et al. (2010) Showed that the spatial distance between the center of mass of a cue and a target alters the magnitude of repulsion. At larger distances, the ARE declines.

Menceloglu, Grabowecky, and Suzuki

Ono and Watanabe
Found that the ARE occurs during interocular transfer, suggesting that repulsion effects in cueing tasks are attentionally driven. This finding is in contrast to DiGiacomo and Pratt (2012), who found no interocular transfer of the ARE.

This study explored the effect of post-cues on attentional repulsion in the visual field. Post-cues distorted spatial representation such that Vernier lines were perceived as appearing closer to the cued location (i.e., attentional attraction). Potential non-attentional factors driving the attraction effect were ruled out in follow up control experiments. 
Petersson et al. (2019) Demonstrated that AREs are not observed for endogenous shifts of attention. A dual-task paradigm was used where participants completed peripheral target detection task on some trials and a centrally presented Vernier alignment task on other trials. Attention was cued via a number presented at fixation, which predicted the likely location of an upcoming peripheral target. While number cues improved response times for target detection (typical cueing effect), no ARE was observed.

Pratt and Arnott (2008) Demonstrated that experimental manipulations that alter chronometric effects in cueing studies have similar effects on the magnitude of the ARE. Different onset and offset cues and color cues were used to shift attention exogenously.

Pratt and Turk-Browne Tested the ARE using guided localization tasks. Here, rather than responding with a keypress, participants moved (2003) a computer mouse or used their limb to make an absolute judgment about the perceived position of an offset line. Typical repulsion effects were observed.

Shim and Cavanagh Studied repulsion effects when attention was deployed to a moving object (as opposed to when it is statically (2005) focused on a single location). Participants were required to focus attention on two bars that moved within a rotating grating while completing an alignment task. Overall, a repulsion was observed, even after controlling for motion and eye movement confounds. 


\begin{abstract}
Suzuki and Cavanagh Original demonstration of the ARE. Peripheral luminance cues that shifted attention altered the perceived position of targets in a centrally presented Vernier alignment task. Single and double cue conditions were tested, as well as predictive and non-predictive cueing tasks. Potential confounds such as visual aftereffects and apparent motion were ruled out in a series of control experiments.
\end{abstract}

Toba, Cavanagh, and Used a line bisection task to demonstrate the ARE. Specifically, participants decided whether a test line bisecting Bartolomeo (2011) a stimulus line appeared to be shifted to the left or right following a cue. Cues appearing on the right-hand side of the screen caused the line to appear shifted leftwards and vice versa.

Yamada, Yuki, and Attraction and repulsion effects were tested using a gaze cueing procedure. Participants were required to determine Kawabe (2013) whether two circles appearing at the cued location were vertically offset. The circles could appear simultaneously with stimulus onset asynchrony (SOA), of $0 \mathrm{~ms}$, or one after the other, with an SOA of $2000 \mathrm{~ms}$. For the $0 \mathrm{~ms}$ SOA, no attraction or repulsion effects were observed. For a $2000 \mathrm{~ms}$ SOA, attentional attraction effects were observed.

Yamada, Yuki, Miura, Mapped the time course of attentional repulsion and attraction effects. Following a cue, participants decided and Kawabe (2011) whether a line appearing in the upper right (or left) corner of a computer screen was aligned to a target appearing in the lower right (or left) corner. The time delay between the appearance of the first target line and the second target line was manipulated. When the time delay was $0 \mathrm{~ms}$, repulsion effects were observed. When the time delay was longer, attraction effects were observed. 


\section{Operationalizing Endogenous and Exogenous Attention}

Before analyzing the ARE literature manipulating attention endogenously, first, it is prudent to establish a clear operationalization of the terms endogenous and exogenous attention. Although endogenous and exogenous attention are commonly used by researchers to refer to voluntary and involuntary processes generally, a brief perusal of the literature reveals that endogenous and exogenous attention have been operationalized in numerous ways, leading to a level of confusion. For example, some have considered the term endogenous attention to be synonymous with the term sustained attention, and the term exogenous attention to be synonymous with transient attention (Carrasco, 2011). However, while exogenous attention is transient, it is not necessarily the case that all transient shifts of attention involve purely exogenous processes. Likewise, although sustained attention likely requires voluntary resources, it can also be maintained using external, environmental cues. Finally, it is important to consider the potential role of eye movements play when understanding visual attention effects. Indeed, attention can be deployed with or without eye movements (referred to as covert and overt attention, respectively). Critically, overt and covert attention can have distinct effects on visual perception (see Kowler, 2011 for a review).

Figures $2 \mathrm{a}$ and $2 \mathrm{~b}$ showcase versions of the Posner Cueing paradigm (Posner, 1980) that we believe are ideal operationalizations of endogenous and exogenous attention. In the endogenous cueing paradigm (Figure 2a), an arbitrary color symbol is presented in central vision, which reliably predicts an upcoming target's location. Following a reasonably long time interval (e.g., $300 \mathrm{~ms}$ or greater), participants complete a target detection or discrimination task either at the cued or un-cued location. A longer time interval is needed for participants to interpret the information provided by the central cue, in turn, reflecting the time course of endogenous attention. 
Furthermore, the cue should only predict the location of the target, not the specific response required from the observer (Carrasco, 2011). Indeed, Carrasco (2011) suggests that a discrimination task that is orthogonal to the cue could be used to ensure that responses remain uncontaminated by decisional biases. Typically, performance is improved for cued compared to un-cued locations for some time (after all, the cue indicates where the target is likely to occur), indicating an attentional shift to the cued location. In contrast, in the exogenous cueing paradigm, cues do not predict the target location. Instead, participants are actively told that any changes in the environment do not predict the target location. Before the target detection task, a brief, nonpredictive luminance cue is presented in the peripheral visual field. For exogenous cueing tasks, the time delay between the cue and target is critical. When the target is presented very soon after the cue (e.g., typically less than $300 \mathrm{~ms}$ ), facilitation is observed at the cued location. However, when there is a slightly longer delay between the cue and the target (e.g., typically greater than 300ms), inhibition is observed (i.e., slower responding at the cued location; Posner, Rafal, Choate, \& Vaughan, 1985).

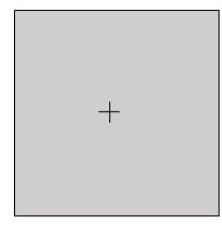

$1000 \mathrm{~ms}$

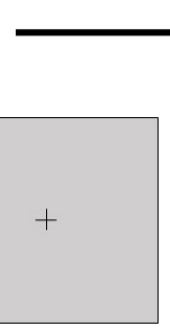

$1000 \mathrm{~ms}$

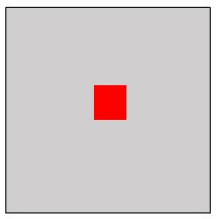

$500 \mathrm{~ms}$ VALID TRIAL



$500 \mathrm{~ms}$ INVALID TRIAL



Press "Space"

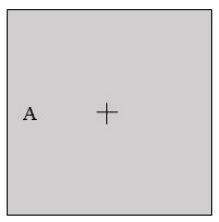

Press "Space"
$=$ target more likely to appear on right side of fixation

$=$ target more likely to appear on left side of fixation 
Figure 2a. Example of an ideal endogenous cueing procedure. Either a red or green cue is presented to participants in central vision. Participants are told that the red cue predicts a target appearing in the right visual field, whereas a green cue predicts a target appearing in the left visual field. Participants should discriminate the target as quickly and accurately as possible.

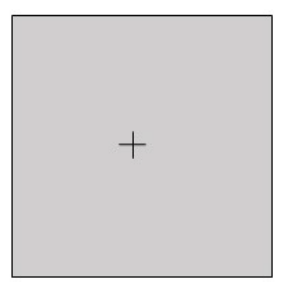

$1000 \mathrm{~ms}$
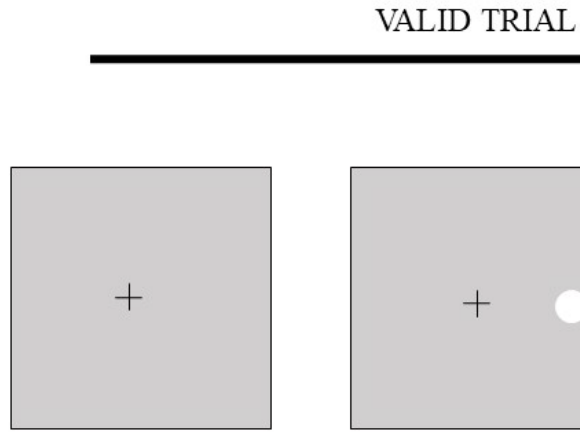

$1000 \mathrm{~ms}$

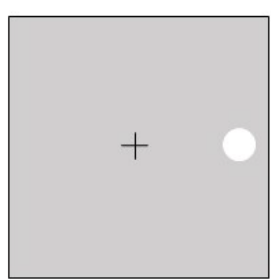

$100 \mathrm{~ms}$ VALID TRIAL

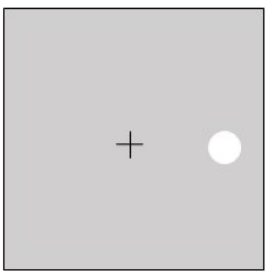

$100 \mathrm{~ms}$

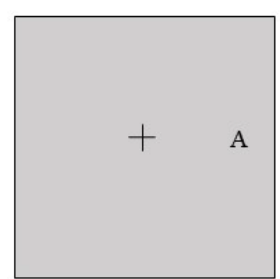

Press "Space"

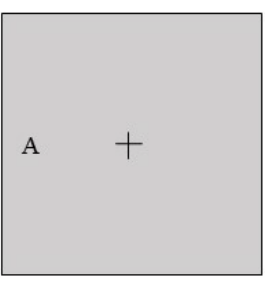

Press "Space"

INVALID TRIAL

Figure $2 b$. Example of an ideal exogenous cueing procedure for measuring attentional facilitation. Briefly presented luminance changes (white circles), automatically pull attention to the cued location. Following this, participants must detect or discriminate a target letter ("A"). The cue is non-predictive, that is, $50 \%$ of trials in an experiment are valid, and $50 \%$ of trials are invalid. 
There are a few key reasons as to why the paradigms described above are ideal for testing endogenous versus exogenous attention. Firstly, in the exogenous paradigm, the cue is nonpredictive and presented in peripheral vision. Furthermore, the time delay between the cue and target is very short. As such, the observer is unable to use any information to push their attention to a particular location based on task goals. Instead, for a shift to occur, attention must be captured by something in the environment and rapidly pulled towards the cued location. In contrast, in the endogenous cueing paradigm, the cue is predictive and presented in central vision. The longer time delay between the cue and target is essential, as the observer must interpret this information and push attention to the relevant location, thus reflecting the sustained time course of endogenous attention. In particular, there is nothing in the environment automatically attracting attention. Nonetheless, these ideal situations are often not employed when testing endogenous and exogenous attention. For example, some researchers have used different sized stimuli, or peripherally presented stimuli to manipulate endogenous (sustained) attention (e.g., Lawrence et al., 2020; Suzuki \& Cavanagh, 1997). However, by definition, anything in the external environment drawing attention to a particular location requires, at least to some extent, exogenous processing. This is because any object suddenly appearing in a peripheral location in the environment is likely to capture attention automatically. Therefore, only central cues, which, when interpreted, allow the observer to push attention towards a relevant location, should be used to study endogenous attention.

Furthermore, as mentioned earlier, researchers have used the terms exogenous and transient interchangeably. This has resulted in researchers using transient but predictive peripheral cues in an attempt to study exogenous attention (e.g., Mounts \& Edwards, 2017; Yeshurun \& Carrasco, 1998, 1999; Yeshurun \& Levy, 2003). While transient cues do tap into the briefer time course of 
exogenous attention, using predictive peripheral cues to manipulate attention may complicate the situation because these types of cues potentially conflate implicit learning and exogenous processes. If attention was being shifted by purely exogenous means, then peripherally presented cues must be considered to be impervious to cue-target contingency learning by participants. Indeed, this is exactly what Giordano et al. (2009) demonstrated in a paper exploring the effect of cue predictability for endogenous versus exogenous attention. Participants were exposed to a central cue or a peripheral cue, followed by a Gabor orientation task. The SOA for the central cue was $300 \mathrm{~ms}$, whereas it was $120 \mathrm{~ms}$ for the exogenous cue, allowing the optimal time course of both processes to be studied. The participant's task was to determine the orientation of one Gabor in the search array (set size 1 or set size 8). Further, participants completed blocks of trials where the validity of the cue varied between $12 \%$ and $100 \%$. Critically, the authors demonstrated that while endogenous cueing effects on target discriminability and processing speed was modulated by cue predictability, exogenous cueing effects remained unaffected by changes in cue-target contingencies.

In contrast, other research on the proportion valid cueing effect suggests that cue predictability can influence the magnitude of cueing effects in peripheral cueing paradigms (Risko \& Stolz, 2010). In this study, participants completed a peripheral cueing task that clearly tapped into the time course of exogenous attention (i.e., 150ms). Critically, 30 participants in the study completed the task with cues that were 50\% valid, whereas another 30 completed the task with cues that were $75 \%$ valid. In contrast to Giordano et al. (2009), the magnitude of the cueing effect was larger in the $75 \%$ valid condition compared to the $50 \%$ valid condition, suggesting that cue validity was contaminating the supposed exogenous nature of the task. However, it is important to note that in this study, only response speeds were affected by cue validity, which may reflect non- 
attentional processes (e.g. Reed, 1973; Risko, Blais, Stolz, \& Benser, 2008). Furthermore, it cannot be said that the cueing effects would be modulated within participants in this study as a between subjects-design was used. Finally, participants were not made explicitly aware of cue validity in this study (see López-Ramón, Chica., Bartolomeo, \& Lupiáñez, 2011 for an exploration of this issue). Thus, while there is debate as to whether cue validity influences purely exogenous processes, we, therefore, suggest that to measure purely exogenous attention, peripherally presented, non-informative cues are the gold standard ideal.

Secondly, it is important to recognize that the endogenous cueing procedure displayed in Figure $2 \mathrm{a}$ uses a completely arbitrary central cue (i.e., two different colored squares). This is in contrast to many studies attempting to manipulate endogenous attention that have employed arrow cues (Chica et al., 2014; Gibson \& Kingstone, 2006). Arrow cues should be avoided when testing endogenous attention because they are overlearned symbols, which have the potential to rapidly shift attention across a broad range of SOAs (Brignani, Guzzon, Marzi, \& Miniussi., 2009; Gibson, Thompson, Davis, \& Biggs, 2011; Gibson \& Bryant, 2005; Guzzon, Brignani, Miniussi, \& Marzi, 2010; Hayward \& Ristic, 2015; Hommel, Pratt, Colzato, \& Godijn, 2001; Ristic, Landry \& Kingstone, 2012; Ristic, Wright \& Kingstone., 2007; Stevens, West, Al-Aidroos, Weger, \& Pratt, 2008). For example, Hommel et al. studied the effect of non-predictive arrows on performance in a target detection task. Here, the idea was that if arrow cues only oriented attention endogenously, then a completely non-predictive arrow presented in central vision should not result in typical orienting effects. However, even when non-predictive arrow cues were used at SOAs as short as $200 \mathrm{~ms}$, attention was oriented. This finding suggests that the overlearned stimuli can rapidly shift attention, which may not involve purely endogenous processes. As such, to test a pure form of endogenous attention, centrally presented stimuli, which are not typically associated with 
particular locations in space, should be used (Brignani et al. 2009; Ristic \& Kingstone, 2006). Here, to effectively complete a cueing task, participants have to interpret the information provided by a central cue consciously, and then orient attention. Note that the participants must be cognizant of the predictability of the cues at the beginning of an experiment; otherwise, cueing effects might be driven by implicitly learned associations (e.g., Lanthier, Wu., Chapman, \& Kingstone, 2015). Using different colored squares such as those shown in Figure 2 is ideal because, at the beginning of the study, the experimenter can tell the participant which location in space is predicted by a particular color, as well as counterbalance the color-location contingencies across participants.

Taken together, although many versions of the Posner cueing paradigm have been used to study spatial attention, some research has failed to test purely exogenous, or purely endogenous attention within a single paradigm, using either peripherally presented predictive cues, or centrally presented directional cues. This conflation has led to a level of confusion in the literature as to how the two types of attentional modes operate. Indeed, as will be demonstrated in the following section, this is undoubtedly the case for studies exploring endogenous attention and the ARE.

\section{Endogenous attention and the Attentional Repulsion Effect}

To date, three studies have explicitly attempted to test the effects of endogenous shifting on repulsion effects (Cutrone et al., 2018; Petersson et al., 2019; Suzuki \& Cavanagh, 1997). Firstly, in their seminal demonstration of the ARE, Suzuki and Cavanagh (1997) used peripheral cues of differing identities to measure voluntary attention effects on performance in a centrally presented Vernier alignment task. Following a variable fixation period, four shapes were presented briefly in each corner of the visual field for $30 \mathrm{~ms}$. The top right and bottom left locations had identical shapes (e.g., squares), and bottom right and top left locations had different identical shapes (e.g., circles). Participants were asked to "attend hard" to one of the two locations indicated 
by the shape types (i.e., two diagonally offset locations) and then hold their attention at these locations for up to $1500 \mathrm{~ms}$, thus requiring sustained attention. Finally, participants completed either a Vernier acuity task or a probe detection task, which was followed by a visual mask. The Vernier task measured the repulsion effect, whereas the probe detection task was included on $50 \%$ of trials as a check to see whether attention was oriented to the valid shape locations (however, the validity of the probe task was at chance, where, on probe trials, the target only fell at the location behind one of the valid shapes on $50 \%$ of trials). Overall, the authors observed an ARE as well as attentional orienting effects whereby reaction times were faster when probes appeared behind valid shape compared to invalid shape locations. Furthermore, in a later experiment, the authors demonstrated that their voluntary and involuntary manipulations of attention produced time-sensitive effects on repulsion. Whereas exogenous attention caused an early, but then declining repulsion effect, the endogenous manipulation resulted in a belated, but more sustained repulsion effect. Together this allowed the authors to conclude that voluntary attention could drive repulsion effects.

Following Suzuki \& Cavanagh's (1997) original demonstration of the ARE, no further studies attempted to explicitly test endogenous attention and repulsion for another 20 years. Recently, however, two studies have appeared addressing this question. The first, Cutrone et al. (2018), used central cues to endogenously manipulate attention. At the beginning of a trial, either two lines that predicted an upcoming target's location, or four neutral lines, were presented in the center of a computer screen around a fixation cross for $300 \mathrm{~ms}$. This longer time course was used so that participants could interpret the centrally presented information, and then endogenously shift attention accordingly. On valid trials, the participants were required to selectively attend to two of four rapid serial visual presentation (RSVP) streams consisting of letters. These RSVP streams 
were presented in each quadrant of the visual field and were presented for $300 \mathrm{~ms}$ to $3000 \mathrm{~ms}$, thus requiring participants to sustain their attention on peripheral locations in the visual field. The participant's task was to detect if the target letter $(\mathrm{X})$ was presented in one of the attended RSVP streams. Critically, the letter X only appeared on a minority of trials. On the majority of trials where the target letter was absent, and following the RSVP stream, participants completed a Vernier alignment task presented in central vision. This task aimed to measure repulsion effects as a result of endogenous attention.

Critically, Cutrone et al. (2018) observed an ARE in the Vernier alignment task, as well as improved performance in the RSVP task for valid compared to neutral cues (i.e., participants had higher target detection rates in the RSVP task when instructed to attend to two locations rather than the four locations in the neutral condition). This indicates that attention was shifted to the regions indicated by the cue and that this had an effect on spatial localization processes. However, while it seems certain that endogenous attention was at work in this study (due to the probabilities in the two-line condition and the time course used), it is also possible that some form of symbolically driven was also at work, due to the directional lines used as cues. Towards this latter point, recall that in the two-line condition, the lines that pointed to potential target locations in the corner of the visual field. As such, they may have acted in a manner similar to arrow cues, which can often generate rapid symbolic shifts of attention (e.g., Hommel et al., 2001). Indeed, the effect of these two lines appeared to be larger than that of a typical exogenous ARE, perhaps because the lines might have engendered both endogenous and symbolic driven attentional processes. Nonetheless, in order to know whether these cues do engender symbolic shifts of attention, they need to be empirically tested at shorter SOAs and be non-predictive in nature. In any case, though, 
it seems most likely that endogenous attention was the largest contributor to the observed ARE in Cutrone et al. (2018).

Finally, in contrast to both Suzuki \& Cavanagh (1997) and Cutrone et al. (2018), Petersson et al. (2019) found endogenous attention to have no repulsion effect. Here, attention was oriented using predictive, centrally presented number cues to orient attention. Again, participants completed a dual-task experiment, which consisted of a target detection task on some trials, and a Vernier alignment task on other trials. Here, participants' attention was cued using two numbers (2 and 5) that were presented for 500ms. Following this, the participants completed either a target discrimination task where targets were presented at cued versus un-cued locations or a Vernier alignment task presented in central vision. Critically, the participants were told that the number 'two' reliably predicted that the peripheral target would appear in the top left-hand location of the screen and that the number 'five' reliably predicted that the peripheral target would appear in the top right location of the screen (this was counterbalanced across participants). Thus, it was assumed that upon seeing the number cues in the experiment, participants would use the information to shift attention to the appropriate location voluntarily. In the peripheral target task, participants had to discriminate the identity of the target $(\mathrm{x}$ or + ) using keys "z" and "/." Surprisingly, Petersson et al. (2019) found no ARE using their endogenous cueing task. This was the case, even though target detection was faster at cued compared to uncued locations.

Why did Petersson et al. (2019) fail to observe a repulsion effect? One possibility posed by Petersson et al. (2019) is that some form of visual stimulation in the peripheral visual field, prior to the onset of the Vernier stimulus in central vision is necessary for observing attentional repulsion. This stimulation could be, for example, the appearance of a cue such as the appearance of the peripheral shapes in Suzuki and Cavanagh (1997) prior to the onset of the Vernier stimulus. 
Alternatively, this visual stimulation could be the appearance of a target to be processed, such as the RSVP stream adopted in Cutrone et al. (2018). In contrast to these studies, on Vernier target trials in Petersson et al. (2019), there was no visual stimulation in the peripheral visual field. Instead, only a number appeared in central vision, and participants had to orient attention to an empty location in space. Nonetheless, it is important to note that peripheral stimulation appears to be insufficient in itself to cause repulsion effects. Recall that in Suzuki and Cavanagh (1997), brief luminance onsets occur at all four locations of the visual, but a sustained effort is used to attend to two of the four shapes. Likewise, participants must pay close attention to two of four offset RSVP streams in Cutrone et al.'s (2018) task. If the ARE was purely stimulus-driven, then ARE effects should not have been observed in these studies, as sensory stimulation was equivalent in all four quadrants of the visual field. However, reliable repulsion effects were observed, suggesting that attention plus sensory stimulation is necessary for the ARE.

Alternatively, it is possible that to observe a repulsion effect in Petersson et al. (2019), all that is needed are markers in the visual field, which indicate potential target locations during a cueing task (Figure 3). That is, rather than having peripheral stimulation in the form of a cue (Suzuki \& Cavanagh, 1997), or a peripheral RSVP target task (Cutrone et al., 2018), markers could be presented at the onset of a trial to indicate potential locations in the visual field that participants could shift attention to. Indeed, in Petersson et al. (2019), the lack of visual markers in the display may have had resulted in participants not knowing exactly where to shift attention to following a cue. In turn, this could have weakened the overall repulsion effect due to differences in the final locus of attention across participants and trials (e.g., attention shifted further away from the intended cued location). This seems likely, as the presence versus absence of placeholder stimuli is known to have qualitatively different effects on chronometric measures of spatial 
attention shifting (e.g. Hilchey, Pratt, \& Christie, 2016; Taylor, Chan, Bennet, \& Pratt, 2015). For instance, Taylor et al. (2015) showed that the spatial distribution of attentional facilitation and inhibition for placeholder present versus absent conditions differed when attention was cued exogenously.
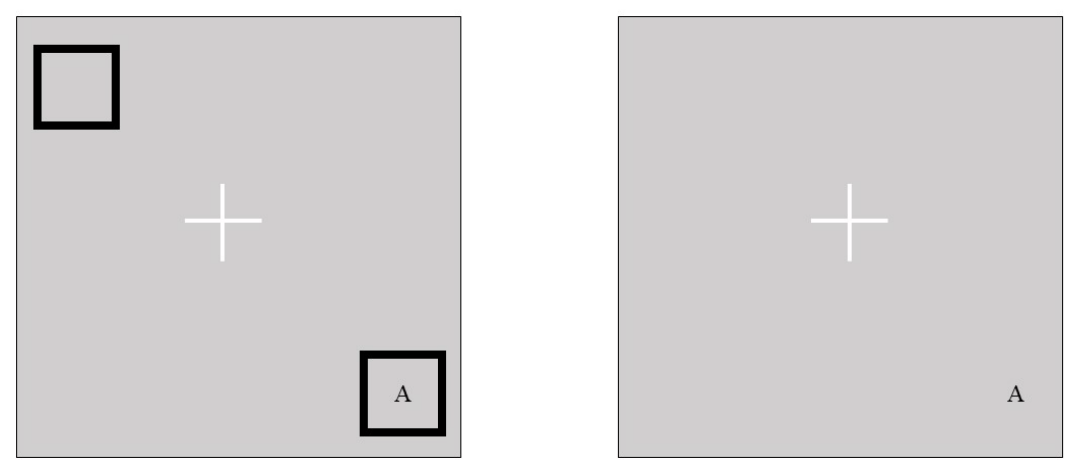

Figure 3. Cueing displays containing placeholders (left panel) or no placeholders (right panel). The participant's task would be to detect the letter A as quickly as possible. Here, the placeholder stimuli are full boxes; however, it is also possible to use corners of boxes (e.g., Ling \& Carrasco, 2006) to avoid placeholders potentially masking target stimuli (e.g., Gibson, 1996, but see Yantis \& Jonides, 1996), or even lines as placeholders - see Figure 4).

Finally, it is possible that the endogenous manipulation utilized by Petersson et al. (2019) simply was not strong enough or the appropriate type with which to observe a repulsion effect. There are a few reasons for this. First, in Petersson and colleague's task, cues directed attention to only one location in the visual field. Given that the SOA between the cue and the target was 500ms, and that the target was to only appear in one location of the visual field, it is highly likely that participants would have shifted their gaze to the peripheral visual field. This would mean that the 
authors were not testing covert attention. In contrast to this, typical studies on the ARE use simultaneous cues that point to two opposite locations in the visual field. Double cueing, as opposed to single cueing, is known to produce more substantial repulsion effects (Suzuki \& Cavanagh, 1997). Furthermore, the use of double cues would likely lower the chance that participants would shift their gaze to a single point in the visual field. Instead, it would be more efficient to center their gaze on fixation to attend to the two cue locations simultaneously. Second, Petersson et al. (2019) used cues that were 55.81\% predictive of target location across both attention probe trials ( $80 \%$ with 360 trials), and vernier ARE trials ( $0 \%$ with 156 trials). Typically, endogenous cueing procedures use highly predictive cues across all conditions so that participants are able to use this information to shift attention accordingly. In contrast to this, the cues used in Peterrson et al. (2019) may have simply not contained enough information across all the trials to encourage participants to use it to shift attention. Nonetheless, this possibility seems unlikely, namely due to the fact that a chronometric cueing effect was observed in the data (albeit a fairly weak one). Finally, even though Petersson et al. (2019) did observe a small reliable difference in reaction time for their peripheral target task, it is possible that these differences in reaction time were driven by differences in processing speed, discriminability, decisional or motor processes (e.g., Carrasco \& McElree, 2001; Dosher, Han, \& Lu, 2004; Reed, 1973; Wickelgren, 1977; Yashar and Lamy, 2011).

Given the uncertainty in the literature, the aim of the current study was to test whether endogenous repulsion could be observed using a paradigm similar to that adopted by Petersson et al. (2019) that included placeholders, as well as a double cueing procedure. We predicted that with these modifications, a reliable ARE would be observed. Specifically, as shown in Figure 4, following a period of fixation, participants were shown either a red or a green circle. These circles 
acted as endogenous cues. After this, participants completed either a peripheral target task or a Vernier acuity task. In the peripheral target task, two shapes appeared at opposite diagonals corners of the computer screen. Participants had to indicate whether the two shapes were the same (i.e., two circles, two squares) or different from one another (i.e., one circle and one square). On $80 \%$ of the trials, the color of the circle predicted the locations of the peripheral targets (i.e., which corners of the screen the two shapes would appear in). Accuracy and reaction time in this task was measured for valid and invalid cues and allowed us to confirm that attention was endogenously shifted in response to the cue. In the Vernier Acuity task, participants viewed two briefly presented lines that appeared above or below fixation. The top line could be parallel, shifted to the left, or shifted to the right of the bottom line. Participants indicated whether they perceived the top line to be oriented to the left or right of the bottom line, and the proportion of "left" responses was calculated for each cue direction to measure the attentional repulsion effect. Specifically, a relatively higher proportion of 'left' responses following a cue to the top right and bottom left of the screen compared to when cued to the top left and bottom right of the screen would indicate a repulsion effect. 
A)

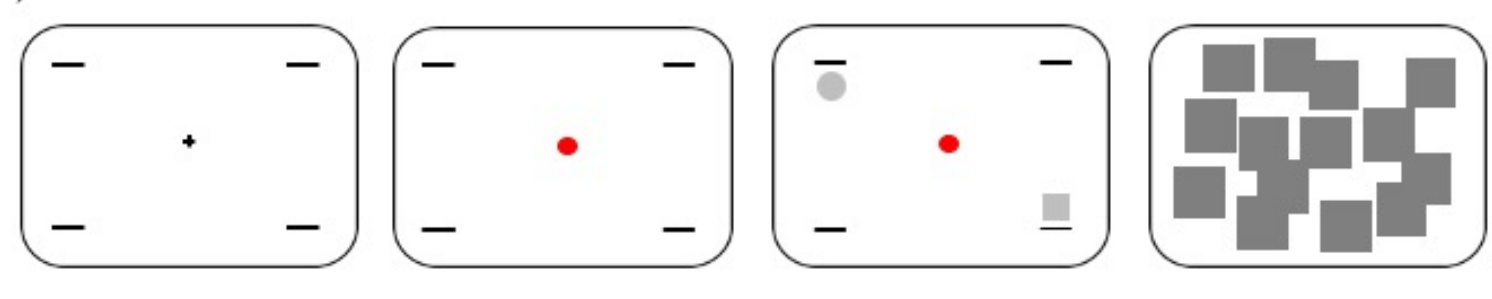

B)

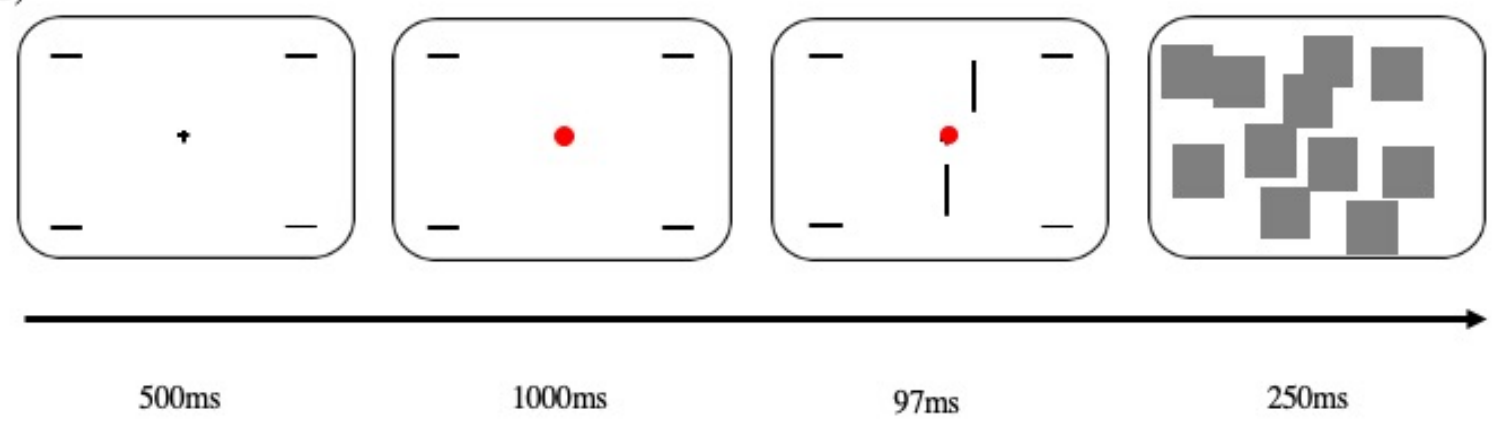

Figure 4. The general method used in the current study. Following a period of fixation, a centrally presented, predictive color cue was shown for $1000 \mathrm{~ms}$. Following this, participants completed either a peripheral target discrimination task (A) or a Vernier Line task (B). 


\section{Method}

\section{Participants}

A priori power analysis using a sample size planner that corrects for publication bias and uncertainty was used to determine the sample size for the current study (Anderson, Kelley, \& Maxwell, 2017). In order to obtain an effect size similar to those observed in Experiment 2 of Petersson et al. (2019) with statistical power of .8 and assurance of .5, a sample size of at least 37 participants is required. As such, we decided to collect data from forty participants for our later analyses. Forty participants from the University of Toronto participated in the current study for course credit or monetary compensation $(\$ 10)$ and gave informed consent. The mean age of the participants was 19.20 years $(S D=2.17)$, with 33 participants identifying as female, and seven as male. Four participants reported being left-handed, and all reported normal or corrected to normal vision.

\section{Stimuli and Apparatus}

An LCD monitor that was $580 \mathrm{~mm}$ by $300 \mathrm{~mm}$ in size, that had a resolution set to 2560 pixels by 1440 pixels, and a refresh rate of $144 \mathrm{~Hz}$ was used to present stimuli. Participants were placed in an artificially lit room with their head placed on a chin rest that was $570 \mathrm{~mm}$ away from the monitor. This meant that 1 degree of visual angle roughly corresponded to 48 pixels. The stimuli were created using the psychophysics toolbox (Brainard,1997) in MATLAB. Stimuli were presented on a black background, which had a luminance of approximately $0 \mathrm{~cd} / \mathrm{m}^{2}$. Throughout the experiment, participants were asked to focus their gaze on a centrally presented white dot that had a luminance $177 \mathrm{~cd} / \mathrm{m}^{2}$ and a radius of. $29^{\circ}$ of visual angle. Four placeholder stimuli were also presented at each corner of the computer screen approximately $10.9^{\circ}$ diagonally from fixation. 
These placeholders were thin grey lines (luminance $=38 \mathrm{~cd} / \mathrm{m}^{2}$ ) that horizontally subtended $1.5^{\circ}$ of visual angle and were 2 pixels in height.

At the beginning of a trial, the fixation point and placeholder stimuli were presented alone for approximately 500ms. Participants were asked to maintain their gaze on this central point, although eye movements were not recorded. ${ }^{1}$ Following this circle, cues were presented centrally. These cues were equal luminance red or green colored circles $\left(35 \mathrm{~cd} / \mathrm{m}^{2}\right)$ that reliably predicted the location of targets in the peripheral target discrimination task. The circles subtended $.5^{\circ}$ of visual angle and were presented for $1000 \mathrm{~ms}$ before the appearance of the targets. This longer cue presentation was chosen to allow participants enough time to interpret the cue and voluntarily shift attention accordingly. For half of the participants, the green cue reliably predicted that the targets in the peripheral discrimination task would appear in the top right and bottom left of the visual field, while the red cue reliably predicted that the target would appear in the top left and bottom right of the visual field. The opposite was true for the remaining participants

Finally, participants completed one of two tasks: a peripheral target task, or a Vernier acuity task. For the peripheral target task, two shapes appeared at the same, or opposite corners of the computer screen to the cue. The shapes were filled with white ovals and rectangles (luminance $=177 \mathrm{~cd} / \mathrm{m}^{2}$ ) that subtended approximately $.8^{\circ}$ of visual angle and were presented for approximately $97 \mathrm{~ms}$. For the Vernier acuity task, two white lines subtending 1.6 were presented $7.7^{\circ}$ above or below fixation for $97 \mathrm{~ms}$ (luminance $=177 \mathrm{~cd} / \mathrm{m}^{2}$ ). The bottom line was always placed on the vertical meridian. On one-third of trials, the top line was also placed on the vertical meridian, on one-third, it was placed $.2^{\circ}$ to the left of the vertical meridian, and on the final third it was placed $.2^{\circ}$ to the right of the vertical meridian. After the presentation of the peripheral discrimination or the Vernier line task, a mask consisting of squares of differing luminance covering the entire screen 
as a grid was presented for $250 \mathrm{~ms}$, followed by a blank screen. For the peripheral discrimination task, the participants reported whether the two shapes presented were the same or different as quickly and accurately as possible by pressing "Z" and "X" on the keyboard. For the Vernier task, participants reported whether the top line was shifted to the left or right of the bottom line by pressing the "<" key or the ">" key, respectively. This was followed by an inter-trial interval of $1300 \mathrm{~ms}$.

\section{Design and Procedure}

A within-subjects design was used, where all participants completed both the Vernier and the peripheral target detection task. Firstly, the participants completed a practice version of the experiment consisting of 32 trials. To proceed to the main experiment, participants had to achieve accuracy of greater than $75 \%$ on the practice block $^{2}$. In the experiment proper, there were 560 trials. On $70 \%$ of trials, participants completed valid peripheral target discrimination trials (i.e., the targets fell at the locations predicted by the cue). On $8.6 \%$ of trials, participants completed invalid peripheral target discrimination trials (i.e., targets fell at the opposite location predicted by the cue. Finally, on the remaining $21.4 \%$ of trials, participants completed the Vernier line task in central vision. Participants were explicitly informed of the predictability of the cues at the beginning of the experiment and told to use this information to complete the task to the best of their ability. This ensured endogenous attention was tested. Rest breaks were offered regularly throughout, and the study took approximately 1 hour to complete. 


\section{Results}

Peripheral Target Discrimination. Firstly, we examined accuracy and reaction time data from valid and invalidly cued trials in the peripheral target task. For the accuracy data, all but one participant performed above chance level (i.e., greater than $60 \%$ for at least one condition), and their data were removed from further analyses ${ }^{3}$. No statistical outliers were detected using Tabachnick \& Fidell (2013) Z-score criterion of +/- 3.29 for detecting outliers. Nonetheless, Shapiro-Wilk tests indicated that the assumption of normality was violated for data from invalidly cued trials $(p<.05)$, so parametric and non-parametric tests were run on the data. A paired samples t-test revealed a non-significant effect of cue validity on accuracy, $t(38)<.001, p=.1 .00, g=0$, as did a Wilcoxon signed ranks test, $Z=.25, p=.801$. Mean accuracy for validly cued trials was $85.85 \%(S D=7.66 \%)$ and was $85.85 \%(S D=10.06 \%)$ for invalidly cued trials.

Next, reaction time data were examined. For each individual case, reaction times for correct responses that were $+/-2$ standard deviations away from the participants mean reaction time were removed. This led to the removal of approximately $4.63 \%(S D=1.67 \%)$ of correct trials per participant from further analysis. Next, the combined group reaction time data were screened for outliers using Tabachnick \& Fidell (2013) Z-score criterion of +/- 3.29, and none were detected. Finally, Shapiro-Wilk tests confirmed that the assumption of normality was met $(p$ 's $>.05)$. Critically, a paired samples t-test revealed that cue validity influenced reaction times in the peripheral discrimination task, $t(38)=-3.14, p=.003, g=.16$, where participants were faster to react following valid cues $(M=493 \mathrm{~ms}, S D=171 \mathrm{~ms})$ compared to invalid cues $(M=521 \mathrm{~ms}, S D=$ $172 \mathrm{~ms})$. This demonstrates that participants were able to use the predictability of the central cues, and endogenously shift attention to opposite corners of the visual field. 
Vernier Task. Next, we analyzed the data from the Vernier acuity task. Initially, we calculated the proportion of trials participants responded that the top line was shifted to the left of the bottom line as a function of cue location and Vernier location (Petersson et al., 2019). If the cue was influencing perceived spatial position, then the proportion of "left" responses should be higher for cues appearing in the top right/bottom left of the screen compared to cues appearing in the top left/bottom right of the screen. After calculating individual proportions, the data were screened for statistical outliers using the +/- 3.29 Z-score cut-off. Although no outliers were detected, normality was violated for the condition where attention was cued to the top right and bottom left of the visual field, and the top Vernier was displaced to the left of bottom Vernier. Furthermore, sphericity was violated. Therefore, parametric and non-parametric tests were run where necessary, and Greenhouse-Geisser corrected values are reported.

As displayed in Figure 5, there was a main effect of cue location on responses, $F(1,38)=$ $13.89, p=.001, \eta \mathrm{p} 2=.268$, where the proportion of 'left' responses was higher following cues to the top right and bottom left of the screen $(M=58.38 \%, S D=16.27 \%)$, compared to cues to the top left and bottom right of the screen $(M=50.64 \%, S D=15.30 \%)$. Furthermore, this was confirmed by a non-parametric Wilcoxon Test comparing the effect of cue location on the proportion of 'left' responses, $Z=-3.41, p=.001$. Together, this provides strong evidence that participants experienced attentional repulsion, and perceived the Vernier line as appearing further away from the cued location than it physically was.

Furthermore, there was a main effect of Vernier line location, $F(1.56,59.13)=94.58, p<$ $.001, \eta p 2=.713$. As depicted in Figure 5, participants had a higher proportion of 'left' responses when the top Vernier appeared to the left of the bottom Vernier $(M=71.79 \%, S D=16.48 \%)$, compared to the middle $(M=55.45 \%, S D=17.25 \%)$ and right Vernier locations $(M=36.28 \%$, 
$S D=17.70 \%$ ). Two Wilcoxon signed ranks tests confirmed this finding $Z$ (left versus middle) $=$ 5.01, $p<.001, Z$ (middle versus right) $=5.04, p<.001$. This shows that participants were able to discriminate between the left and right locations of the Vernier, regardless of the location of the cue. Finally, there was no interaction between the cue location and the Vernier location, $F(2,76)$ $=2.53, p=.086, \eta p 2=.062$.



- Top left and bottom right location cued

Figure 5. The proportion of "Left" responses made in the Vernier acuity task, as a function of cue and Vernier location. Within-subject error bars shown (Cousineau, 2005) 


\section{Discussion}

One of the most striking consequences of spatially shifting attention is the ARE, where observers experience distortions in the perception of space at unattended locations following a shift of attention. Nonetheless, only three studies have explicitly tested the effects of endogenous attention on repulsion, with conflicting results (Cutrone et al., 2018; Suzuki \& Cavanagh, 1997; Petersson et al., 2019). For instance, using centrally predictive cues, Petersson et al. (2019) found typical cueing effects but failed to observe an ARE. Nonetheless, this null finding may have been a consequence of the particular design adopted by the authors, who used single rather than double cues to orient attention and did not include placeholders in their visual display. As such, the aim of the current study was to test whether endogenous ARE could be observed using a purely endogenous manipulation of attention. Specifically, spatial attention was directed to two opposite corners of the visual field using a centrally presented double cueing procedure. Furthermore, placeholders were included in the visual display so that participants knew in advance the exact locations in the visual field to shift attention to. This ensured that conditions were made as favorable as possible for observing an ARE. The effect of this manipulation on vision was tested using both a chronometric measure of attention, as well as a Vernier acuity task.

Critically, using these optimal conditions, we observed both typical chronometric effects and spatial repulsion effects. Firstly, reaction times for the discrimination of targets appearing in the peripheral visual field were faster following valid compared to invalid cues. This suggests that attention was shifted as a consequence of our centrally presented, informative cues (for alternate interpretations see Carrasco \& McElree, 2001; Reed, 1973; Wickelgren, 1977; Dosher, Han, \& Lu, 2004; Yashar \& Lamy, 2011). Secondly, these central cues also caused distortions in the 
perception of space in the Vernier task. Specifically, when attention was cued to the top left and bottom right of the visual field, participants perceived the top Vernier line as being shifted to the right. Likewise, when attention was cued to the top right and bottom left of the visual field, the top line was perceived as being shifted to the left. Thus, it appears that when using an optimal cueing procedure, endogenous attention does indeed result in attentional repulsion.

The results of the current study suggest that placeholders may be critical in observing endogenous ARE. Indeed, it is likely that the placeholders indicated to participants the exact location where attention needed to be shifted for optimal performance in the peripheral target task, and thus, strengthened repulsion effects in central vision. Indeed, Taylor et al. (2015) found that placeholders markedly influenced the distribution of facilitation and inhibition in a large scale cueing experiment. Here, attention was cued to one of four locations in the visual field using either placeholder present or absent displays. Following this, attentional targets could appear at one of 121 possible locations. Critically, the authors found that the distribution of attention around the cued location was far more restricted in the placeholder present condition, suggesting that attention was specifically directed to the locations indicated by the placeholders (for similar results see Hilchey, Pratt \& Christie, 2016; Jefferies \& Di Lollo, 2017; 2014). Alternatively, rather than using placeholders, all that may be needed is for observers to tightly focus attention in space, perhaps by imagining a small dot in the visual field with which to deploy cognitive resources ${ }^{4}$. In any case, the current results provide converging evidence with these studies suggesting that the presence of placeholders result in qualitatively different patterns of results when it comes to visual perception and attention.

Furthermore, our results suggest that double-cueing procedures may make it more likely that endogenous repulsion effects are observed. Indeed, in contrast to Petersson et al. (2019), both 
Cutrone et al. (2018), and the current study all used double cues to orient attention. As shown in Figure 6, double cues might cause larger repulsion effects, as double cues would hypothetically shift more receptive fields away from the central visual field. In turn, this would allow for greater repulsion effects in a Vernier alignment task. Furthermore, a recent study has demonstrated that AREs are most potent when the cue and Vernier appear in the same half of the computer screen (Baumeler \& Born, 2019). Compared to single cues, double cues ensure that a cue and Vernier are always present in each hemifield, thus allowing Verniers to be dragged away from one another, and thus would strengthen attentional repulsion effects.

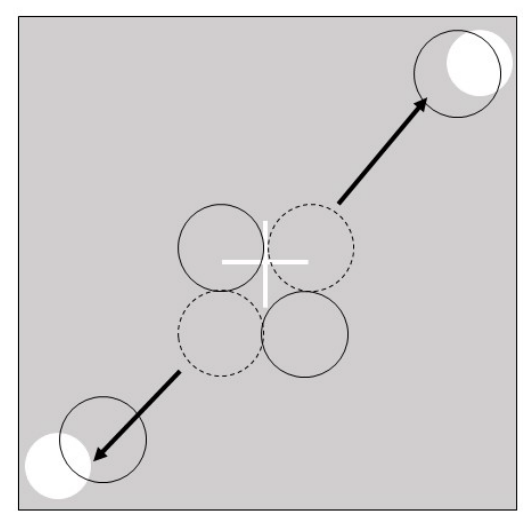

Double Cue Condition



Single Cue Condition

Figure 6. A hypothetical example of receptive fields shifting following a single versus double cue in the ARE paradigm. The arrows depicted in the Figure represent the direction of the shift in receptive fields when attention is deployed. As indicated by the arrows, in the double cue conditions, the receptive fields shift to the top right and bottom left of the screen. In the single cue condition, the receptive field shifts to the top right of the screen only. 
Finally, although the focus of the current paper has been on understanding the relationship between endogenous cueing and the ARE, it is essential to recognize that these are not the only ways in which attention is directed in the visual environment. Instead of considering endogenous and exogenous attention as the only methods with which to engender attentional repulsion effects, research shows that there are a number of different ways in which attention might be shifted, and thus, may also cause repulsion effects. For example, the influence of selection history (Awh, Belopolsky, \& Theeuwes, 2012) is yet to be fully explored using the ARE paradigm. 


\section{Footnotes.}

1. Although eye movements were not recorded in the current study, it is important to note that eye movements would have been unlikely to occur in this task. This is because, on each trial, two targets would appear at opposite corners of the visual field simultaneously, or in central vision. It would have therefore been detrimental for participants to shift their eyes in response to the cue as they could only move their eyes to one, and not both of the locations. This would likely lower performance to chance level.

2. Two participants completed the practice block four times and failed to achieve an accuracy of greater than $75 \%$. Nonetheless, they completed the experimental block and performed the peripheral cueing task above chance (i.e., greater than $60 \%$ in at least one condition). As such, their data were included in our main analyses.

3. In an earlier version of this manuscript, data from all forty participants was erroneously included in analysis. Specifically, data from one participant had been reverse scored due to what was believed to be below reverse responding (i.e. performance consistently below chance). However, it was later realized that the participant performed below chance due to using invalid response keys rather than reverse responding. Thus, their reverse scored data has been removed from the final analysis presented in this manuscript.

4. We thank an anonymous reviewer for this suggestion. 
Acknowledgments: This work was supported by an NSERC Grant (2016-06359) awarded to Jay Pratt.

Disclosure Statement: The authors declare no conflicts of interest associated with the research, and that the research meets the ethical guidelines, including adherence to the legal requirements of the study country

Open Science Framework Link to Data:

https://osf.io/fqb56/?view_only=cfa8d2389c3d41a6a082c6c2f34b70eb

Open Science Framework DOI: 10.17605/OSF.IO/FQB56 


\section{References}

Anderson, S. F., Kelley, K., \& Maxwell, S. E. (2017). Sample-size planning for more accurate statistical power: A method adjusting sample effect sizes for publication bias and uncertainty. Psychological science, 28(11), 1547-1562. https://doi.org/ $10.1177 / 0956797617723724$

Anton-Erxleben, K., \& Carrasco, M. (2013). Attentional enhancement of spatial resolution: Linking behavioural and neurophysiological evidence. Nature Reviews Neuroscience, 14(3), 188-200. https://doi.org/10.1038/nrn3443

Anton-Erxleben, K., Stephan, V. M., \& Treue, S. (2009). Attention reshapes center-surround receptive field structure in macaque cortical area MT. Cerebral Cortex, bhp002.

Arnott, S. R., \& Goodale, M. A. (2006). Distorting visual space with sound. Vision Research, 46(10), 1553-1558. https://doi.org/10.1016/j.visres.2005.11.020

Au, R. K. C., Ono, F., \& Watanabe, K. (2013). Spatial distortion induced by imperceptible visual stimuli. Consciousness and Cognition, 22(1), 99-110. https://doi.org/10.1016/j.concog.2012.11.010

Awh, E., Belopolsky, A. V., \& Theeuwes, J. (2012). Top-down versus bottom-up attentional control: A failed theoretical dichotomy. Trends in Cognitive Sciences, 16(8), 437-443. https://doi.org/10.1016/j.tics.2012.06.010

Barbot, A., Landy, M. S., \& Carrasco, M. (2012). Differential effects of exogenous and endogenous attention on second-order texture contrast sensitivity. Journal of Vision, 12(8), 6-6. https://doi.org/10.1167/12.8.6 
Baumeler, D., \& Born, S. (2019). Vertical and horizontal meridian modulations suggest areas with quadrantic representations as neural locus of the attentional repulsion effect. Journal of Vision, 19(6), 15-15. https://doi.org/10.1167/19.6.15

Brainard, D. H. (1997). The psychophysics toolbox. Spatial vision, 10(4), 433-436. https://doi.org/ $10.1163 / 156856897 \times 00357$

Brignani, D., Guzzon, D., Marzi, C. A., \& Miniussi, C. (2009). Attentional orienting induced by arrows and eye-gaze compared with an endogenous cue. Neuropsychologia, 47(2), 370381. https://doi.org/10.1016/j.neuropsychologia.2008.09.011

Carrasco, M. (2011). Visual attention: The past 25 years. Vision Research, 51(13), 1484-1525. https://doi.org/ 10.1016/j.visres.2011.04.012

Carrasco, M., Barbot A. (2015). How attention affects spatial resolution. Cold Spring Harbor Symposia on Quantitative Biology, 149-160. http://dx.doi.org/10.1101/sqb.2014.79.024687.

Carrasco, M., \& McElree, B. (2001). Covert attention accelerates the rate of visual information processing. Proceedings of the National Academy of Sciences, 98(9), 5363-5367. https://doi.org/ 10.1073/pnas.081074098

Carrasco, M., \& Yeshurun, Y. (2009). Covert attention effects on spatial resolution. Progress in Brain Research, 176, 65-86. https://doi.org/10.1016/S0079-6123(09)17605-7

Chica, A. B., Martín-Arévalo, E., Botta, F., \& Lupiáñez, J. (2014). The Spatial Orienting paradigm: How to design and interpret spatial attention experiments. Neuroscience \& Biobehavioral Reviews, 40, 35-51. https://doi.org/10.1016/j.neubiorev.2014.01.002 
Chien, S., Ono, F., \& Watanabe, K. (2011). Mislocalization of Visual Stimuli: Independent Effects of Static and Dynamic Attention. PLOS ONE, 6(12), e28371. https://doi.org/10.1371/journal.pone.0028371

Connor, C. E., Gallant, J. L., Preddie, D. C., \& Van Essen, D. C. (1996). Responses in area V4 depend on the spatial relationship between stimulus and attention. Journal of Neurophysiology, 75(3), 1306-1308. https://doi.org/10.1152/jn.1996.75.3.1306

Corbetta, M., \& Shulman, G. L. (2002). Control of goal-directed and stimulus-driven attention in the brain. Nature reviews neuroscience, 3(3), 201-215. https://doi.org/10.1038/nrn755

Cousineau, D. (2005). Confidence intervals in within-subject designs: A simpler solution to Loftus and Masson"s method. Tutorials in Quantitative Methods for Psychology, 1(1), 42-45. https://doi.org/10.20982/tqmp.01.1.p042

Cutrone, E. K., Heeger, D. J., \& Carrasco, M. (2018). On spatial attention and its field size on the repulsion effect. Journal of Vision, 18(6), 8-8. https://doi.org/10.1167/18.6.8

de Haas, B., Schwarzkopf, D. S., Anderson, E. J., \& Rees, G. (2014). Perceptual load affects spatial tuning of neuronal populations in human early visual cortex. Current Biology, 24(2), R66R67. https://doi.org/10.1016/j.cub.2013.11.061

Desimone, R., \& Duncan, J. (1995). Neural mechanisms of selective visual attention. Annual Review of Neuroscience, 18(1), 193-222.

DiGiacomo, A., \& Pratt, J. (2012). Misperceiving space following shifts of attention: Determining the locus of the attentional repulsion effect. Vision Research, 64, 35-41. https://doi.org/10.1016/j.visres.2012.05.009 
Dosher, B. A., Han, S., \& Lu, Z. L. (2004). Parallel processing in visual search asymmetry. Journal of Experimental Psychology: Human Perception and Performance, 30(1), 3-27. https://doi.org/10.1037/0096-1523.30.1.3

Dugué, L., Merriam, E. P., Heeger, D. J., \& Carrasco, M. (2018). Specific visual subregions of TPJ mediate reorienting of spatial attention. Cerebral Cortex, 28(7), 2375-2390. https://doi.org/ 10.1093/cercor/bhx 140

Fischer, J., \& Whitney, D. (2009). Attention Narrows Position Tuning of Population Responses in V1. Current Biology, 19(16), 1356-1361. https://doi.org/10.1016/j.cub.2009.06.059

Folk, C. L., Remington, R. W., \& Johnston, J. C. (1992). Involuntary covert orienting is contingent on attentional control settings. Journal of Experimental Psychology: Human Perception and Performance, 18(4), 1030-1044. https://doi.org/10.1037/0096-1523.18.4.1030

Gibson, B. S. (1996). Visual quality and attentional capture: A challenge to the special role of abrupt onsets. Journal of Experimental Psychology: Human Perception and Performance, 22(6), 1496-1504. https://doi.org/10.1037/0096-1523.22.6.1496

Gibson, B. S., \& Bryant, T. A. (2005). Variation in cue duration reveals top-down modulation of involuntary orienting to uninformative symbolic cues. Perception \& psychophysics, 67(5), 749-758. https://doi.org//10.3758/BF03193530

Gibson, B. S., \& Kingstone, A. (2006). Visual attention and the semantics of space: Beyond central and peripheral cues. Psychological Science, 17(7), 622-627. https://doi.org/ $\underline{10.1111 / j .1467-9280.2006 .01754 . x}$

Gibson, B. S., Thompson, A. N., Davis, G. J., \& Biggs, A. T. (2011). Going the distance: Extrasymbolic contributions to the symbolic control of spatial attention. Visual Cognition, 19(10), 1237-1261. https://doi.org/10.1080/13506285.2011.628636 
Giordano, A. M., McElree, B., \& Carrasco, M. (2009). On the automaticity and flexibility of covert attention: A speed-accuracy trade-off analysis. Journal of Vision, 9(3), 30-30. https://doi.org/10.1167/9.3.30

Goodale, M. A., \& Milner, A. D. (1992). Separate visual pathways for perception and action. Trends in Neurosciences, 15(1), 20-25.

Gozli, D. G., \& Pratt, J. (2012). Attentional repulsion effect despite a colour-based control set. Visual Cognition, 20(6), 696-716. https://doi.org/10.1080/13506285.2012.683051

Grubb, M. A., White, A. L., Heeger, D. J., \& Carrasco, M. (2015). Interactions between voluntary and involuntary attention modulate the quality and temporal dynamics of visual processing. Psychonomic Bulletin \& Review, 22(2), 437-444. https://doi.org/10.3758/s13423-014$\underline{0698-y}$

Guzzon, D., Brignani, D., Miniussi, C., \& Marzi, C. A. (2010). Orienting of attention with eye and arrow cues and the effect of overtraining. Acta Psychologica, 134(3), 353-362. https://doi.org/10.1016/j.actpsy.2010.03.008

Hayward, D. A., \& Ristic, J. (2015). Exposing the cuing task: The case of gaze and arrow cues. Attention, Perception, \& Psychophysics, 77(4), 1088-1104.

https://doi.org/10.3758/s13414-015-0877-6

Hein, E., Rolke, B., \& Ulrich, R. (2006). Visual attention and temporal discrimination: Differential effects of automatic and voluntary cueing. Visual Cognition, 13(1), 29-50. https://doi.org/10.1080/13506280500143524

Hilchey, M. D., Pratt, J., \& Christie, J. (2016). Placeholders dissociate two forms of inhibition of return. The Quarterly Journal of Experimental Psychology, 1-13. 
Hommel, B., Pratt, J., Colzato, L., \& Godijn, R. (2001). Symbolic control of visual attention. Psychological Science, 12(5), 360-365. https://doi.org/10.1111/1467-9280.00367

Hopf, J.-M., Boehler, C. N., Luck, S. J., Tsotsos, J. K., Heinze, H.-J., \& Schoenfeld, M. A. (2006). Direct neurophysiological evidence for spatial suppression surrounding the focus of attention in vision. Proceedings of the National Academy of Sciences, 103(4), 1053-1058. https://doi.org/10.1073/pnas.0507746103

Hopfinger, J. B., \& West, V. M. (2006). Interactions between endogenous and exogenous attention on cortical visual $\quad$ processing. NeuroImage, 31(2), $\quad$ 774-789. https://doi.org/10.1016/j.neuroimage.2005.12.049

Hubel, D. H., \& Wiesel, T. N. (1962). Receptive fields, binocular interaction and functional architecture in the cat's visual cortex. Journal of Physiology (London), 160(1), 106-154. https://doi.org/10.1113/jphysiol.1962.sp006837

Hubel, D. H., \& Wiesel, T. N. (1968). Receptive fields and functional architecture of monkey striate cortex. The Journal of Physiology, 195(1), 215-243. Retrieved from https://www.ncbi.nlm.nih.gov/pmc/articles/PMC1557912/

Jefferies, L. N., \& Di Lollo, V. (2017). Deployment of spatial attention to a structural framework: exogenous (alerting) and endogenous (goal-directed) factors. Attention, Perception, \& Psychophysics, 79(7), 1933-1944. https://.do.org/ 10.3758/s13414-017-1378-6

Jefferies, L. N., Enns, J. T., \& Di Lollo, V. (2014). The flexible focus: Whether spatial attention is unitary or divided depends on observer goals. Journal of Experimental Psychology: Human Perception and Performance, 40(2), 465-470. https://.doi.org/10.1037/a0034734

Jonides, J. (1981). Voluntary versus automatic control over the mind's eye's movement. Attention and Performance, 187-203. Retrieved from https://ci.nii.ac.jp/naid/20001365380/ 
Kincade, J. M., Abrams, R. A., Astafiev, S. V., Shulman, G. L., \& Corbetta, M. (2005). An eventrelated functional magnetic resonance imaging study of voluntary and stimulus-driven orienting of attention. Journal of Neuroscience, 25(18), 4593-4604. https://doi.org/10.1523/JNEUROSCI.0236-05.2005

Klein, B. P., Paffen, C. L. E., Pas, S. F. te, \& Dumoulin, S. O. (2016). Predicting bias in perceived position using attention field models. Journal of Vision, 16(7), 15-15. https://doi.org/10.1167/16.7.15

Kosovicheva, A. A., Fortenbaugh, F. C., \& Robertson, L. C. (2010). Where does attention go when it moves?: Spatial properties and locus of the attentional repulsion effect. Journal of Vision, 10(12), 33-33. https://doi.org/10.1167/10.12.33

Kowler, E. (2011). Eye movements: The past 25 years. Vision Research, 51(13), 1457-1483. https://doi.org/10.1016/j.visres.2010.12.014

Lanthier, S. N., Wu, D. W.-L., Chapman, C. S., \& Kingstone, A. (2015). Resolving the controversy of the proportion validity effect: Volitional attention is not required, but may have an effect. Attention, Perception, \& Psychophysics, 77(8), 2611-2621. https://doi.org/10.3758/s13414-015-0956-8

Lawrence, R. K., Edwards, M., \& Goodhew, S. C. (2020). The impact of scaling rather than shaping attention: Changes in the scale of attention using global motion inducers influence both spatial and temporal acuity. Journal of Experimental Psychology: Human Perception and Performance, 46(3), 313-323. https://doi.org/10.1037/xhp0000708

Ling, S., \& Carrasco, M. (2006). When sustained attention impairs perception. Nature Neuroscience, $9(10), 1243-1245$. https://doi.org/10.1038/nn1761 
López-Ramón, M. F., Chica, A. B., Bartolomeo, P., \& Lupiáñez, J. (2011). Attentional orienting and awareness: Evidence from a discrimination task. Consciousness and cognition, 20(3), 745-755. https://doi.org/10.1016/j.concog.2010.10.024

Macaluso, E., \& Doricchi, F. (2013). Attention and predictions: control of spatial attention beyond the endogenous-exogenous dichotomy. Frontiers in Human Neuroscience, 7, 685. https://doi.org/10.3389/fnhum.2013.00685

Menceloglu, M., Grabowecky, M., \& Suzuki, S. (2018). Near complete interocular transfer of the attentional repulsion effect. Vision Research, 150, 24-28. https://doi.org/10.1016/j.visres.2018.06.011

Mounts, J. R., \& Edwards, A. A. (2017). Attentional breadth and trade-offs in spatial and temporal acuity. Visual Cognition, 1-12.

Müller, H. J., \& Rabbitt, P. M. (1989). Reflexive and voluntary orienting of visual attention: Time course of activation and resistance to interruption. Journal of Experimental Psychology. Human Perception and Performance, 15(2), 315-330. https://doi.org/10.1037//00961523.15.2.315

Nakayama, K., \& Mackeben, M. (1989). Sustained and transient components of focal visual attention. Vision Research, 29(11), 1631-1647. https://doi.org/10.1016/00426989(89)90144-2

Ono, F., \& Watanabe, K. (2011). Attention Can Retrospectively Distort Visual Space. Psychological Science, 22(4), 472-477. https://doi.org/10.1177/0956797611403319

Peelen, M. V., Heslenfeld, D. J., \& Theeuwes, J. (2004). Endogenous and exogenous attention shifts are mediated by the same large-scale neural network. Neuroimage, 22(2), 822-830. https://doi.org/ 10.1016/j.neuroimage.2004.01.044 
Petersson, A. M., Hilchey, M. D., \& Pratt, J. (2019). Examining the Role of Attention and Sensory Stimulation in the Attentional Repulsion Effect. Frontiers in Psychology, 10. https://doi.org/10.3389/fpsyg.2019.00238

Posner, M. I. (1980). Orienting of attention. Quarterly Journal of Experimental Psychology, 32(1), $3-25$.

Posner, M. I., \& Cohen, Y. (1984). Components of visual orienting. Attention and Performance X: Control of Language Processes, 32, 531-556.

Posner, M. I., Rafal, R. D., Choate, L. S., \& Vaughan, J. (1985). Inhibition of return: Neural basis and function. Cognitive Neuropsychology, 2(3), 211-228.

Pratt, J., \& Arnott, S. R. (2008). Modulating the attentional repulsion effect. Acta Psychologica, 127(1), 137-145. https://doi.org/10.1016/j.actpsy.2007.03.003

Pratt, J., \& Turk-Browne, N. B. (2003). The attentional repulsion effect in perception and action. Experimental Brain Research, 152(3), 376-382. https://doi.org/10.1007/s00221-003$1557-7$

Prinzmetal, W., McCool, C., \& Park, S. (2005). Attention: Reaction Time and Accuracy Reveal Different Mechanisms. Journal of Experimental Psychology: General, 134(1), 73-92. https://doi.org/10.1037/0096-3445.134.1.73

Reed, A. V. (1973). Speed-accuracy trade-off in recognition memory. Science, 181(4099), 574576. https://doi.org/ 10.1126/science.181.4099.574

Risko, E. F., Blais, C., Stolz, J. A., \& Besner, D. (2008). Covert orienting: A compound-cue account of the proportion cued effect. Psychonomic Bulletin \& Review, 15(1), 123-127. https://doi.org/10.3758/PBR.15.1.123 
Risko, E. F., \& Stolz, J. A. (2010). The proportion valid effect in covert orienting: Strategic control or implicit learning? Consciousness and Cognition, 19(1), 432-442. https://doi.org/10.1016/j.concog.2009.07.013

Ristic, J., \& Kingstone, A. (2006). Attention to arrows: Pointing to a new direction. The Quarterly Journal of Experimental Psychology, 59(11), 1921-1930.

Ristic, J., Landry, M., \& Kingstone, A. (2012). Automated Symbolic Orienting: The Missing Link. Frontiers in Psychology, 3. https://doi.org/10.3389/fpsyg.2012.00560

Ristic, J., Wright, A., \& Kingstone, A. (2007). Attentional control and reflexive orienting to gaze and arrow cues. Psychonomic Bulletin \& Review, 14(5), 964-969. https://doi.org/10.3758/BF03194129

Shim, W. M., \& Cavanagh, P. (2005). Attentive tracking shifts the perceived location of a nearby flash. Vision Research, 45(25), 3253-3261. https://doi.org/10.1016/j.visres.2005.05.029

Stevens, S. A., West, G. L., Al-Aidroos, N., Weger, U. W., \& Pratt, J. (2008). Testing whether gaze cues and arrow cues produce reflexive or volitional shifts of attention. Psychonomic Bulletin \& Review, 15(6), 1148-1153. https://doi.org/10.3758/PBR.15.6.1148

Suzuki, S., \& Cavanagh, P. (1997). Focused attention distorts visual space: An attentional repulsion effect. Journal of Experimental Psychology: Human Perception and Performance, 23(2), 443-463. https://doi.org/10.1037/0096-1523.23.2.443

Tabachnick, B. G., \& Fidell, L. S. (2013). Using multivariate statistics: International edition. Pearson2012.

Taylor, J. E. T., Chan, D., Bennett, P. J., \& Pratt, J. (2015). Attentional cartography: mapping the distribution of attention across time and space. Attention, Perception, \& Psychophysics, 77(7), 2240-2246. https://doi.org/10.3758/s13414-015-0943-0. 
Toba, M.-N., Cavanagh, P., \& Bartolomeo, P. (2011). Attention biases the perceived midpoint of horizontal lines. $\quad$ Neuropsychologia, $\quad 49(2), \quad 238-246$. https://doi.org/10.1016/j.neuropsychologia.2010.11.022

Wickelgren, W. A. (1977). Speed-accuracy tradeoff and information processing dynamics. Acta Psychologica, 4l(1), 67-85. https://doi.org/10.1016/0001-6918(77)90012-9

Wiesel, T. N., \& Hubel, D. H. (1966). Spatial and chromatic interactions in the lateral geniculate body of the rhesus monkey. Journal of Neurophysiology, 29(6), 1115-1156. https://doi.org/10.1152/jn.1966.29.6.1115

Womelsdorf, T., Anton-Erxleben, K., Pieper, F., \& Treue, S. (2006). Dynamic shifts of visual receptive fields in cortical area MT by spatial attention. Nature Neuroscience, 9(9), 11561160. https://doi.org/10.1038/nn1748

Yamada, Yuki, \& Kawabe, T. (2013). Gaze-cueing of attention distorts visual space. Universitas $\begin{array}{llll}\text { Psychologica, } & \text { 12(SPE5), } & \text { 1505-1514. } & \text { Retrieved }\end{array}$ http://www.scielo.org.co/scielo.php?script=sci_abstract\&pid=S165792672013000500008\&lng=en\&nrm=iso\&tlng=pt

Yamada, Yuki, Miura, K., \& Kawabe, T. (2011). Temporal course of position shift for a peripheral target. Journal of Vision, 11(6), 6-6. https://doi.org/10.1167/11.6.6

Yantis, S., \& Jonides, J. (1996). Attentional capture by abrupt onsets: New perceptual objects or visual masking? Journal of Experimental Psychology: Human Perception and Performance, 22(6), 1505-1513. https://doi.org/10.1037/0096-1523.22.6.1505 
Yashar, A., \& Lamy, D. (2011). Refining the dual-stage account of intertrial feature priming: Does motor response or response feature matter?. Attention, Perception, \& Psychophysics, 73(7), 2160. https://doi.org//10.3758/s13414-011-0182-y

Yeshurun, Y., \& Carrasco, M. (1998). Attention improves or impairs visual performance by enhancing spatial resolution. Nature, 396(6706). https://doi.org/10.1038/23936

Yeshurun, Y., \& Carrasco, M. (1999). Spatial attention improves performance in spatial resolution. Vision Research, 39(2), 293-306. https://doi.org/10.1016/S0042-6989(98)00114-X

Yeshurun, Y., \& Levy, L. (2003). Transient spatial attention degrades temporal resolution. Psychological Science, 14(3), 225-231.

Yeshurun, Y., Montagna, B., \& Carrasco, M. (2008). On the flexibility of sustained attention and its effects on a texture segmentation task. Vision Research, 48(1), 80-95. https://doi.org/10.1016/j.visres.2007.10.015

Zeki, S. (1980). The representation of colours. Nature, 284, 413. 